\title{
INVERSE LIMITS AND MULTICOHERENCE
}

\author{
BY SAM B. NADLER, JR.
}

\section{Communicated by Leonard Gillman, August 19, 1969}

1. Introduction. In this paper we state some results concerning inverse limits and multicoherence and give applications to hyperspaces and inverse limits of special types of spaces. The proofs of these and other related results will appear elsewhere.

By a metric continuum we mean a nonempty compact connected metric space. A space $X$ is said to have property (b) (see [2, p. 63] or $[10$, p. 226]) if and only if, given a continuous function $f$ from $X$ into the unit circle in the plane, there exists a continuous real valued function $\alpha$ defined on $X$ such that $f(x)=e^{i \alpha(x)}$ for each $x \in X$. If $X$ is a metric continuum, then we say that $X$ has multicoherence degree $k$ (see [3] or [10, p. 83]) provided l.u.b. $\left\{r\left(X_{1}, X_{2}\right): X_{1}\right.$ and $X_{2}$ are subcontinua of $X$ with $\left.X_{1} \cup X_{2}=X\right\}=k$, where $r\left(X_{1}, X_{2}\right)$ denotes one less than the number of components of $X_{1} \cap X_{2}$. The multicoherence degree of $X$ is denoted by $r(X)$. We note that $r(X)=0$ is equivalent to $X$ being unicoherent. It is well known that if $X$ is a metric continuum with property (b), then $X$ is unicoherent (see $[2$, p. 69] or $[10$, p. 227$])$, but not conversely.

All inverse systems considered in this paper are countable and the inverse limit of an inverse sequence $\left\{X_{i}, f_{i}\right\}_{i=1}^{\infty}$ is denoted by proj lim $\left\{X_{i}, f_{i}\right\}_{i=1}^{\infty}$. For notation and terminology relating to inverse limits, see $[1]$.

\section{Basic theorems.}

THEOREM 1. If $X=\operatorname{proj} \lim \left\{X_{i}, f_{i}\right\}_{i=1}^{\infty}$ and each space $X_{i}$ is a metric continuum with property (b), then $X$ has property (b).

Theorem 2. If $X=$ proj $\lim \left\{X_{i}, f_{i}\right\}_{i=1}^{\infty}$ where, for each $i=1,2$, $\cdots, X_{i}$ is a metric continuum, $r\left(X_{i}\right) \leqq k$, and $f_{i}$ is a mapping of $X_{i+1}$ onto $X_{i}$, then $r(X) \leqq k$.

We note that if each of the bonding maps $f_{i}$ in Theorem 2 are monotone and $r\left(X_{i}\right)=k$ for all $i=1,2, \cdots$, then $r(X)=k$. This observation leads to very simple proofs of Theorem 4.8 and Theorem 4.11 of $[1]$.

AMS Subject Classifications. Primary 5455; Secondary 5425.

Key Words and Phrases. Dendrite, dendroid, inverse limit, monotone map, multicoherence, property (b), unicoherence. 
CoRollary 1. If $X=\operatorname{proj} \lim \left\{X_{i}, f_{i}\right\}_{i=1}^{\infty}$ where $X_{i}$ is a unicoherent metric continuum and $f_{i}$ maps $X_{i+1}$ onto $X_{i}$ for each $i=1,2, \cdots$, then $X$ is unicoherent.

Corollary 2. Let $X=\operatorname{proj} \lim \left\{X_{i}, f_{i}\right\}_{i=1}^{\infty}$ where each space $X_{i}$ is a compact metric space. If every subcontinuum of $X_{i}$ has property (b) (has multicoherence degree $\leqq k$ ) for all $i=1,2, \cdots$, then every subcontinuum of $X$ has property (b) (has multicoherence degree $\leqq k$ ).

Theorem 1 states that property (b) is preserved by taking inverse limits (with no restrictions on the bonding maps). Since property (b) is equivalent to unicoherence for locally connected metric continua (see [2] or [10, p. 228]), it follows from Theorem 1 that the inverse limit of locally connected unicoherent metric continua is unicoherent (note that the inverse limit space might not be locally connected). However, in general, an inverse limit of unicoherent metric continua need not be unicoherent. The following example illustrates this.

EXAMPLE. For each $i=1,2, \cdots$, let $X_{i}=S^{1} \cup \mathcal{S}$ where $S^{1}$ is the unit circle in the plane and $\delta=\left\{((t+1) / t) e^{2 \pi i t}: 1 \leqq t<\infty\right\}$. Also, for each $i=1,2, \cdots$, let $r_{i}: X_{i+1} \rightarrow X_{i}$ be given by $r_{i}(z)=z /|z|$ for all $z \in X_{i+1}$. It is easy to verify that the space proj $\lim \left\{X_{i}, r_{i}\right\}_{i=1}^{\infty}$ is homeomorphic to $S^{1}$ but that each space $X_{i}$ is unicoherent.

3. Applications. In this section we state some results on hyperspaces and inverse limits of special types of continua. Their proofs utilize material in the previous section.

Let $\mathbf{2}^{X}=\{K: K$ is a nonempty compact subset of $X\}$ and let $\boldsymbol{C}(\boldsymbol{X})$ $=\left\{K: K \in 2^{X}\right.$ and $K$ is connected $\}$. The topology for $2^{X}$ is the finite topology [7] $\left(C(X)\right.$, as a subset of $2^{x}$ carries the relative topology from $2^{X}$ ). Known theorems (see, for example, [5], [6], [9], or [11]) indicate a general pattern that these hyperspaces are less pathological than the space. Our next theorem shows that if $X$ is any metric continuum, then $2^{X}$ and $C(X)$ have property (b) and therefore are unicoherent. Of course, if $X$ is locally connected, this is a simple consequence of the result in [11].

Theorem 3. If $X$ is a metric continuum, then both $2^{X}$ and $C(X)$ have property (b).

A dendroid is an arcwise connected metric continuum such that each subcontinuum is unicoherent. The following corollary extends Lemma 3 of [8] which states that if a metric continuum admits a continuous selection [7] on its hyperspace of (nonempty) subcontinua, then it is a dendroid.

COROLlary 3. If a metric continuum $X$ is a retract of $C(X)$ (we 
consider $X$ contained in $C(X)$ as the subspace of singletons), then $X$ is arcwise connected and has property (b).

REMARK. It is clear that if $X$ is a locally connected metric continuum, then $X$ is a retract of $2^{X}$ (or of $C(X)$ ) if and only if $X$ is an absolute retract. We pose the following question.

QUESTION. What are necessary and sufficient conditions in order that a metric continuum $X$ be a retract of $2^{X}$ or of $C(X)$ ?

A dendrite $[10, \mathrm{p} .88]$ is a locally connected metric continuum which contains no simple closed curve. A mapping $f: Y \rightarrow Z$ is said to be monotone if and only if $f^{-1}(f(y))$ is a continuum for each $y \in Y$ (see $[10$, p. 70$]$ but note that we do not require $f$ to be onto $Z$ ).

Theorem 4. Let $X=$ proj $\lim \left\{D_{i}, f_{i}\right\}_{i=1}^{\infty}$ where $D_{i}$ is a dendroid for each $i=1,2, \cdots$.

1. If $X$ is arcwise connected, then $X$ is a dendroid.

2. If $X$ is locally connected, then $X$ is a dendrite.

3. If $D_{i}$ is a dendrite and $f_{i}$ is a monotone mapping of $D_{i+1}$ onto $D_{i}$ for each $i=1,2, \cdots$, then $X$ is a dendrite.

Some of the results in [4] are simple consequences of the theorems in this paper and some can be extended using these theorems. The next corollary extends the theorem in [4] which states that if the inverse limit (with onto bonding maps) of arcs is locally connected, then it is an arc.

Corollary 4. If $X=\operatorname{proj} \lim \left\{A_{i}, f_{i}\right\}_{i=1}^{\infty}$ where $A_{i}$ is an arc for each $i=1,2, \cdots$ and if $X$ is arcwise connected, then $X$ is an arc or $a$ singleton.

Proof. By Theorem $4, X$ is a dendroid. If $X$ is neither an arc nor a singleton, then $X$ contains a triod $T$. Using 2.8 of $[1$, p. 235] we see that $T=\operatorname{proj} \lim \left\{\pi_{i}(T), f_{i} \mid \pi_{i+1}(T)\right\}_{i=1}^{\infty}$. Since $\pi_{i}(T)$ is an arc for each $i=1,2, \cdots$, we have a contradiction.

The results in $\$ 2$ can be done in the setting of inverse systems over directed sets and general Hausdorff continua. Using this more general setting, Theorem 3 of $\$ 3$ can be proved for $X$ compact, connected, and Hausdorff.

It has been pointed out to me by Professor John Isbell that property (b) is equivalent to $H^{\prime}(X, Z)=0$ so that Theorem 1 is a consequence of the continuity of Cech theory.

\section{REFERENCES}

1. C. E. Capel, Inverse limit spaces, Duke Math. J. 21(1954), 233-245. MR 15, 976.

2. S. Eilenberg, Transformations continues en circonférence et la topologie du plan, Fund. Math. 26(1936), 61-112. 
3. - Sur les espaces multicohérents. I, Fund. Math. 27(1936), 153-190.

4. M. K. Fort, Jr. and J. Segal, Local connectedness of inverse limit spaces, Duke Math. J. 28(1961), 253-259. MR 23 \#A3549.

5. N. R. Gray, A note on hyperspaces and the Hilbert cube, Topology Conference (Arizona State University, Tempe, Ariz., 1967) Arizona State Univ., Tempe, Ariz., 1968, pp. 147-152.

6. J. L. Kelley, Hyperspaces of a continuum, Trans. Amer. Math. Soc. 52(1942), 22-36. MR 3, 315.

7. E. Michael, Topologies on spaces of subsets, Trans. Amer. Math. Soc. 71(1951), 152-182. MR 13, 54.

8. S. B. Nadler, Jr. and L. E. Ward, Jr., Concerning continuous selections, Proc. Amer. Math. Soc.

9. J. Segal, Hyperspaces of the inverse limit space, Proc. Amer. Math. Soc. 10(1959), 706-709. MR 21 \#7492.

10. G. T. Whyburn, Analytic topology, Amer. Math. Soc. Colloq. Publ., vol. 28, Amer. Math. Soc., Providence, R. I., 1942. MR 4, 86.

11. M. Wojdysławski, Rétractes absolus et hyperespaces des continus, Fund. Math. 32(1939), 184-192.

Dalhousie University, Halifax, Nova Scotia, Canada 